\title{
Alphaherpesvirinae and Gammaherpesvirinae glycoprotein L and CMV UL130 originate from chemokines
}

\author{
Maja Malkowska ${ }^{1}$, Katarzyna Kokoszynska', Magdalena Dymecka ${ }^{1,2}$, Leszek Rychlewski ${ }^{2}$ and Lucjan S Wyrwicz ${ }^{{ }^{*}}$
}

\begin{abstract}
Herpesviridae is a large family of DNA viruses divided into three subfamilies: Alpha-, Beta- and Gammaherpesvirinae. The process of herpesvirus transmission is mediated by a range of proteins, one of which is glycoprotein $\mathrm{L}(\mathrm{gL})$. Based on our analysis of the solved structures of HSV2 and EBV $\mathrm{gH} / \mathrm{gL}$ complexes, we propose that Alphaherpesvirinae and Gammaherpesvirinae glycoprotein $L$ and Betaherpesvirinae UL130 originate from chemokines. Herpes simplex virus type $2 \mathrm{gL}$ and human cytomegalovirus homolog (UL130) adopt a novel C chemokine-like fold, while Epstein-Barr virus gL mimics a CC chemokine structure. Hence, it is possible that gL interface with specific chemokine receptors during the transmission of Herpesviridae. We conclude that the further understanding of the function of viral chemokine-like proteins in Herpesviridae infection may lead to development of novel prophylactic and therapeutic treatment.
\end{abstract}

Keywords: Herpesviridae, Glycoprotein L, GL, UL130, Chemokines, HCMV, HSV2, EBV

\section{Background}

As a family of dsDNA viruses, Herpesviridae are known to infect vertebrates, including humans, and cause progression of various contagious diseases, such as orofacial herpes (HSV1), genital herpes (HSV2), chickenpox and shingles (VZV), opportunistic infections (CMV), Kaposi's sarcoma (KSHV) and mononucleosis (EBV) [1]. Members of the Herpesviridae family have been classified into three distinct subfamilies: Alpha- (HSV1, HSV2, VZV); Beta- (CMV, HHV-6, HHV-7); Gammaherpesvirinae (EBV, KSHV).

The cell-to-cell transmission of virions is one of limiting steps in spread of infection. The targeting of a specific tissue for infection is based on the recognition of definite entry receptors [2]. The virus used as a model of herpetic infection is HSV1. In its genome, 11 out of 80 genes encode outer membrane glycoproteins. According to previous studies, glycoprotein $\mathrm{D}(\mathrm{gD})$, glycoprotein $\mathrm{B}(\mathrm{gB})$ and the complex of glycoproteins $\mathrm{H}$ and $\mathrm{L}(\mathrm{gH} / \mathrm{gL})$ are curial proteins for cell-to-cell fusion [3,4]. Although numerous

\footnotetext{
* Correspondence: lucjan@bioinfo.pl

1 Laboratory of Bioinformatics and Biostatistics, Maria Sklodowska-Curie Memorial Cancer Center and Institute of Oncology, WK Roentgena 5, 02-781, Warsaw, Poland

Full list of author information is available at the end of the article
}

studies on $\mathrm{gH} / \mathrm{gL}$ biology have been carried out, there is still no unequivocal evidence whether this complex plays role in the recognition of specific cells (tissues) or directly takes part in membrane fusion [5].

The chemokine family has been created based on structural similarity. The characteristics features are the Greek key shape and conserved cysteine residues forming SS-bonds and stabilizing the structure. Chemokine family differs in terms of sequence similarity, which vary from less than $20 \%$ to over $90 \%$ [6]. Based on the position of conserved cysteines chemokine classification divides them into four sub-families (CC, CXC, $\mathrm{C}$ and $\mathrm{CX}_{3} \mathrm{C}$ ).

Chemokine mimicry is a common phenomenon among herpesvirus, poxvirus and retrovirus families [7-9]. So far, over 30 chemokine and chemokine receptor mimics have been described. Except for HCMV UL130 and HSV2 gL no other viral proteins have been designated as $\mathrm{C}$ type chemokine homologs [10-12].

\section{Presentation of the hypothesis}

In our previous report, based on bioinformatic analysis of protein families, we concluded the possibility of a distant homology between Herpesviridae glycoprotein L (gL) and chemokines [12]. We also remarked that human

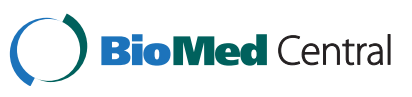

(c) 2013 Malkowska et al.; licensee BioMed Central Ltd. This is an Open Access article distributed under the terms of the Creative Commons Attribution License (http://creativecommons.org/licenses/by/2.0), which permits unrestricted use, distribution, and reproduction in any medium, provided the original work is properly cited. 
Cytomegalovirus (HCMV) contains an additional gene (UL130) which might encode an orthologous chemokinelike protein. This study was followed by crystallographic determination of the structure of two gH/gL complexes. Chowdary and co-workers crystallographically determined the structure of the HSV2 (Herpes simplex virus 2) gH/gL complex, whilst Matsuura et al. described the crystal structure of an Epstein-Barr virus (EBV) complex [13,14].

Although not noticed in the original reports by Chowdary et al. and Matsuura et al., it is important to note that the topologies of both solved glycoproteins are highly concordant with the homology models [12].

We hypothesize that the betaherpevirus UL130 as well as alpha- and gammaherpesvirus gL proteins exhibit structural similarity with chemokines and may interact with their receptors.

The structural comparison between EBV gL and CC-type chemokines leaves no doubt that it adopts a $\mathrm{CC}$ chemokine-like fold. Figure 1 shows the superimposed structures of a CC-type chemokine (human C-C motif chemokine 5; Protein Data Bank entry: 1U4L_A) and the native structure of EBV glycoprotein L (Protein Data Bank entry 3PHF_B) which exhibit a root mean square deviation (RMSD) for C-alpha atoms of $1.23 \AA$ (analysis performed for atoms forming the curved betasheet). The conserved topology is a result of the presence of two cysteine bridges (see Figure 1). Furthermore, the putative interaction surface of EBV's glycoprotein L with chemokines is also preserved (for the conserved pattern of hydrophobic amino acids see Figure 2). The major difference is a result of the altered angle between the helix and the curved beta-sheet, which is to be expected, since the helix in chemokines is involved in dimerization during receptor recognition, whilst gL lacks the ability to dimerize. A further difference can be seen in the presence of two additional helices at the C-terminus of glycoprotein L - 15 and 6 residues long respectively, separated by a link of only two amino acids.

Comparison of cysteine positions in CXC motif in interleukine-8 (IL-8) and lymphotactin (C type chemokine) has shown the conservation of $1^{\text {nd }}$ and $4^{\text {th }}$ cysteine (Figure 3), whereas in case of IL-8 and gL from HSV2 the $1^{\text {st }}$ and $3^{\text {rd }}$ cysteines are conserved (Figure 4). Despite the non-conserved cysteine position, based on structural similarity, we propose that gL HSV2 shall be classified as $\mathrm{C}$ type chemokine-like protein.

Due to synteny, rat cytomegalovirus r131 gene encoding a proinflammatory CC chemokine-like protein has been recognized as HCMV UL130 homolog $[15,16]$. Sequence identity and similarity between r131 and UL130 is $19.1 \%$ and $41.1 \%$ respectively (calculations were performed with PAM250 scoring matrix for global alignment). The position of conserved cysteines $\left(1^{\text {st }}\right.$ and $3^{\text {rd }}$ cysteine of characteristic $\mathrm{CXC}$ type chemokine motif) leads to the assignment of

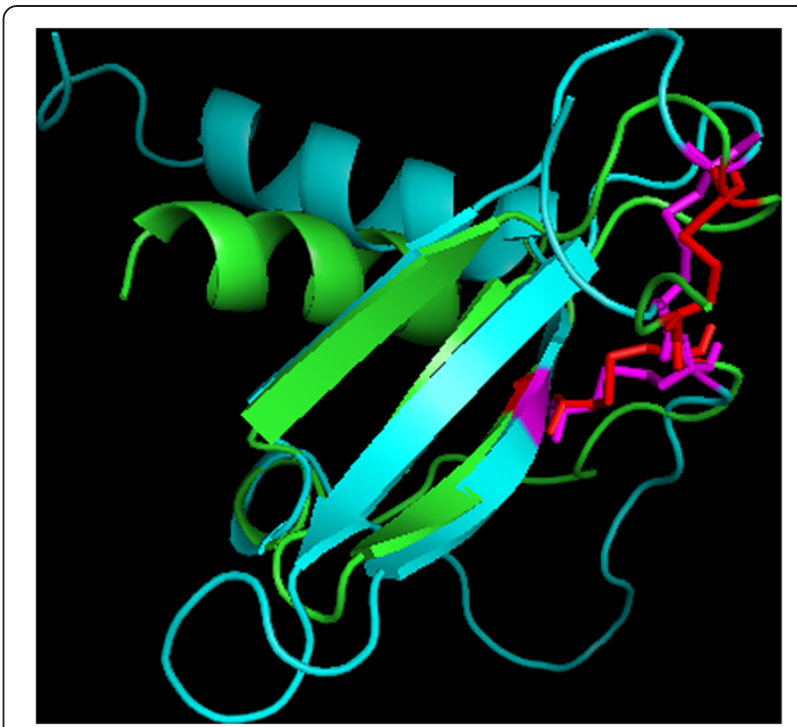

Figure 1 Superimposed native structures of $\mathrm{C}-\mathrm{C}$ motif chemokine 5 (green; PDB entry: 1U4L A) and EBV glycoprotein $\mathbf{L}$ (cyan; PDB entry 3PHF B). The cysteine residues are marked in red ( $C-C$ motif chemokine 5 ) and magenta ( $\mathrm{LL})$.

UL130 to C type chemokine subfamily (Figure 5). Despite of the loss of $2^{\text {nd }}$ and $4^{\text {th }}$ cytosine the chemotactic activity might be preserved and requires further investigation as it might explain the mechanism of UL130 functioning in Endothelial Cell infection [17]

Mutagenesis of interleukine- 8 showed that perturbation of the cysteine bridge between $2^{\text {nd }}$ and $4^{\text {th }}$ motif element had small effects on its function whereas the modification of the disulfide between $1^{\text {st }}$ and $3^{\text {rd }}$ cysteine dramatically reduced potency [18]. This fact supports our hypothesis

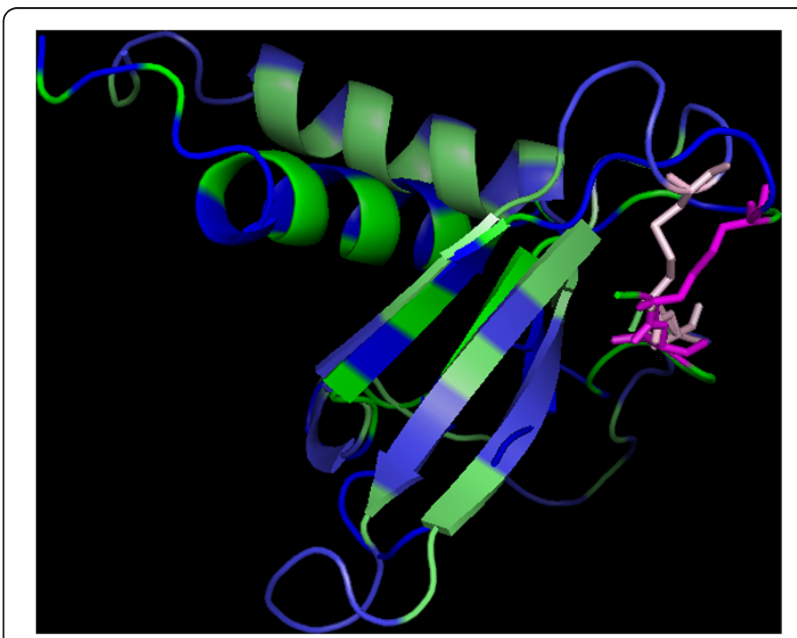

Figure 2 Superimposed native structures of the CC-type chemokine eotaxin-2 (green; PDB entry: 1EIG A) and EBV glycoprotein L (light-green; PDB entry 3PHF B). The hydrophobic surfaces are marked in blue (eotaxin-2) and light-blue (gL), cysteine residues in magenta (eotaxin-2) and light-pink ( $\mathrm{gL}$ ). 


\begin{tabular}{|c|c|c|}
\hline$p d b$ & lymphotactin Homo sapiens & $-\mathrm{HHHHHHHHHHHHH}$ \\
\hline 159162541 & lymphotactin Homo sapiens | 1J8I_A & (5) VSD RTIVSLTTQRL--PVSRIFTYTITEGSLR-- \\
\hline 114565221 & lymphotactin Pan troglodytes & TYTITEGSLR----A \\
\hline 296229862 & lymphotactin-like Callithrix jacchus & VRI TYTIKEGSLK----AVIFIT \\
\hline 19705461 & lymphotactin Rattus norvegicus & ZKI TYTIKE GAMR-----AVIFVT \\
\hline psipred & Iymphotactin Rattus norvegicus & $-\overline{E E} E E E E-------\overline{E E E E E}=$ \\
\hline 149708061 & lymphotactin-like Equus caballus & RKAVESVDFTTRR (90) \\
\hline 291397464 & lymphotactin-like oryctolagus cuniculus & TAIESISN PST (90) \\
\hline 57164253 & lymphotactin Ovis aries & TYTIKEGSMK----AVIFIT \\
\hline$p d b$ & IL8 Homo sapiens & - \\
\hline 10834978 & IL8 Homo sapiens|1IL8 & CIKTYSKPFHP FIRELRVIESGPHCANTEIIVKLSD-GRELCLDPENWVQRVVEKFLRAEN (71) \\
\hline 45384324 & IL8 Gallus gallus & NVNLTPSGPHCKNVEV: \\
\hline 227432980 & IL8 Pelodiscus sinensis & NVKL TQQSGPHCQNVEI IATL D-GREV \\
\hline 300810957 & IL8 Pagrus major & KVELIPANSHCEETEI IATL KRTQ \\
\hline psipred & IL8 Pagrus major & EEE---- HHHHHHHHHHHH--- \\
\hline 17907787 & IL8 Triakis scyllium & LNPEAAWVK KITDMIIINSEK (98) \\
\hline 57163815 & IL8 Felis catus & EIFLKKAEK (98) \\
\hline 88702702 & IL8 Columba livia & PTAPWVQRIVNATMA AQL (97) \\
\hline \multicolumn{3}{|c|}{$\begin{array}{l}\text { Figure } 3 \text { Multiple sequence alignment of lymphotactin (XCL1) and interleukin-8 (IL-8). The corresponding sequences were labeled with } \\
\text { their GenBank entries (denoted by the GenBank identifier - gi) and organism of origin. The numbers in brackets refer to the positions of the } \\
\text { presented sequence fragments. The observed (Protein Data Bank entries } 1 \mathrm{~J} 8 \mathrm{I} \text { A A for XCL1 and } 1 \mathrm{IL} 8 \text { _A for IL-8) and predicted (Psipred) secondary } \\
\text { structure elements are coded with letters ( } \mathrm{H} \text { - a-helix, G-310 helix, E- b-strands). Disulphide bridges in IL-8 has been marked with arrows } \\
\text { and numbered. }\end{array}$} \\
\hline
\end{tabular}

that partial conservation of cysteines in UL130 does not necessarily imply a lack of chemotactic activity.

The speculation concerning the co-evolution of the two interacting glycoproteins [14] causes us to presume that the most likely cause of such concordance is that glycoprotein $\mathrm{L}$ arose as a result of lateral gene transfer from the host. It is very likely that early in the evolution of Herpesviridae, glycoprotein L acted predominantly as a virus encoded chemokine (compare viral chemokines: UL146, UL147, UL152 encoded by HCMV [19]), which later co-evolved with the other membrane viral genes.

In presented analysis we have used a typical homology modeling workbench. The sets of homologs from the NR database (NCBI; National Center for Biotechnology) were created using PSI-BLAST [20] search method and aligned with ClustalW [21]. Secondary structure predictions for selected proteins were calculated using PSIPRED method
[22]. Alignment figures were made with the use of BioEdit version 7.1.3 alignment editor [23]. Residue conservation were presented in alignment with the following default for the software scheme: positively charged highlighted in red; negatively charged in blue; hydrophobic in dark green; tryptophan in light green; polar uncharged and proline in gray; histidine in magenta; glycine in yellow; alanine, phenylalanine and tyrosine in cyan; cysteine in maroon. Modeller 9 was used to create homology models [24]. The structures were visualized with PyMOL v. 0.99rc6 which also enables RMSD calculations [25].

\section{Testing the hypothesis}

Very low sequence similarity between various chemokines and gL prevents any phylogenetic studies of the origin of these protein families from being carried out, and we are unable to justify whether the different topology types

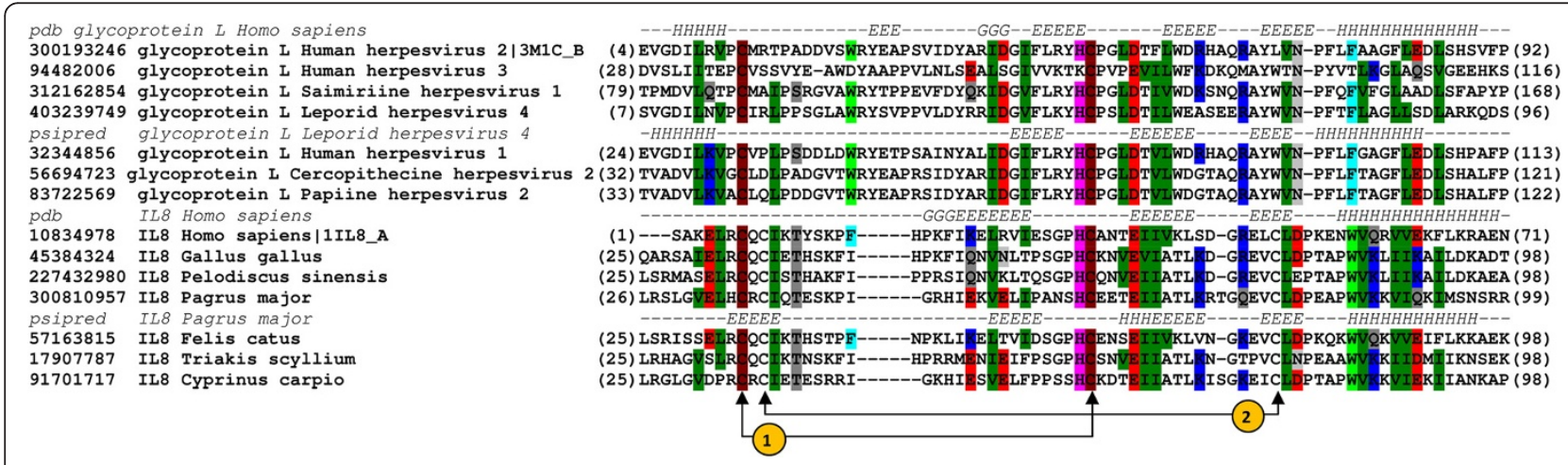

Figure 4 Multiple sequence alignment of glycoprotein L (found in Alphaherpesvirinae) and interleukin-8 (IL-8). The corresponding sequences were labeled with their GenBank entries (denoted by the GenBank identifier - gi) and organism of origin. The numbers in brackets refer to the positions of the presented sequence fragments. The observed (Protein Data Bank entries 3M1C_B for gL and 1IL8_A for IL-8) and predicted (Psipred) secondary structure elements are coded with letters ( $\mathrm{H}$ - a-helix, $\mathrm{G}-3_{10}$ helix, E - b-strands). Disulphide bridges in IL-8 has been marked with arrows and numbered. 


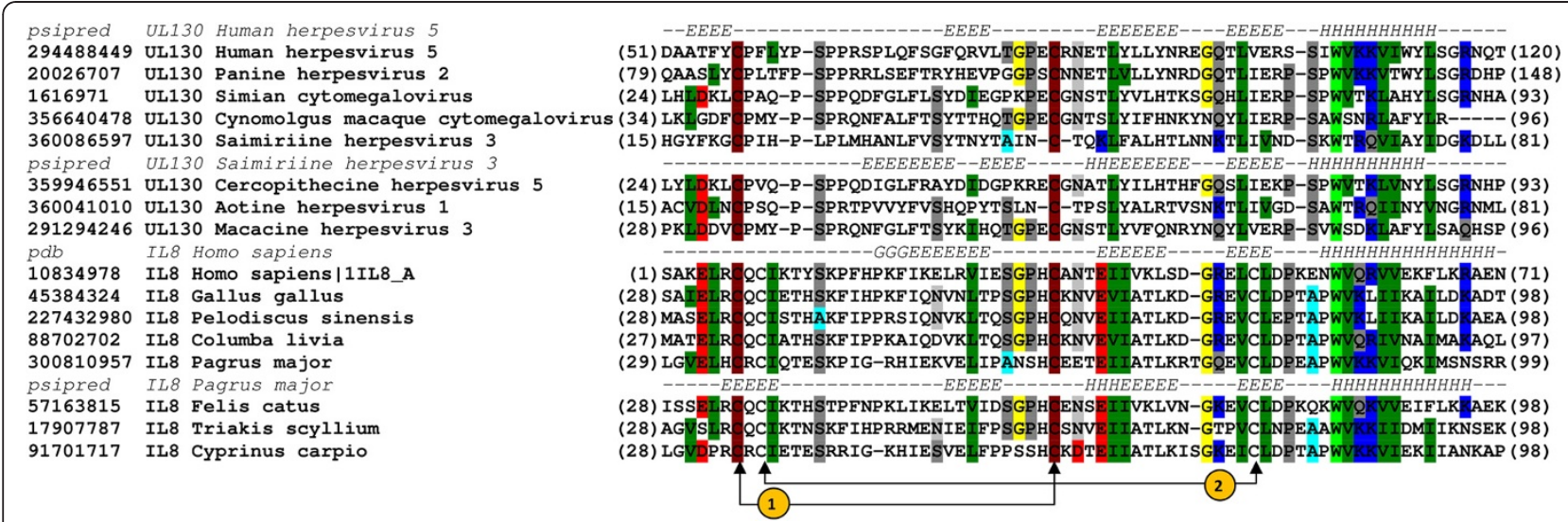

Figure 5 Multiple sequence alignment of UL130 (found in Betaherpesvirinae) and interleukin-8 (IL-8). The corresponding sequences were labeled with their GenBank entries (denoted by the GenBank identifier - gi) and organism of origin. The numbers in brackets refer to the positions of the presented sequence fragments. The observed (Protein Data Bank entry 1IL8_A for IL-8) and predicted (Psipred) secondary structure elements are coded with letters ( $\mathrm{H}$ - a-helix, G-310 helix, E - b-strands). Disulphide bridges in IL-8 has been marked with arrows and numbered.

(CC for Gammaherpesvirinae, $\mathrm{C}$ for the Alpha group and HCMV) come from a single event in the early divergence of Herpesviridae, or rather arose from two independent events of lateral gene transfer.

Discussed here glycoproteins are present in viral envelope and mediate virus entry to host cells. Their mechanism and function as chemokine-like proteins however, remains unknown. There is a potential for further analysis by experimental characterization of specific chemotactic activities of gL and UL130 proteins. Necessary step would be in vivo expression during viral persistence and verification of their release from cells. To establish if these proteins are functional chemokines and which cells are their targets in vivo migration assays shall be performed. Site-directed mutagenesis would help to determine functional domains that are critical for migration. Ligand-binding assays could indicate binding affinity to chemokine receptors.

\section{Implications of the hypothesis}

We conclude that gL could interact with specific cellular chemokine receptors during the invasion of Herpesviridae. The results of our study lead to the conclusion that the herpesvirus transmission process can be dependent on gL interaction with specific cellular chemokine receptors. The presented hypothesis might become a basis for creating new vaccine targets as well as binding inhibitors, which will contribute to the development of novel therapeutic and prophylactic strategies. However, all the assumptions require further studies [26,27]. In order to understand the role of gL in infection and answer questions about its exact functioning, further study is required. The analogical role of UL130 in HCMV and its role in infection needs further explanation.
Competing interests

The authors declare that they have no competing interests.

\section{Authors' contributions}

MM, KK, LSW participated in the design of the study. MM, KK, MD, LR and LSW carried out the bioinformatics analysis. MM, KK, LSW wrote the manuscript. All authors read and approved the final manuscript.

\section{Acknowledgements}

This work was supported by the Polish National Centre for Research and Development (NCBiR) INNOTECH Programme (IN1/159417).

\section{Author details}

'Laboratory of Bioinformatics and Biostatistics, Maria Sklodowska-Curie Memorial Cancer Center and Institute of Oncology, WK Roentgena 5, 02-781, Warsaw, Poland. BiolnfoBank Institute, Limanowskiego 24A, 60-744, Poznań, Poland.

Received: 6 April 2012 Accepted: 18 December 2012

Published: 2 January 2013

\section{References}

1. Whitley RJ: Herpesviruses. In Medical Microbiology. 4th edition. Edited by Baron S. Galveston (TX): 1996

2. Spear PG: Herpes simplex virus: receptors and ligands for cell entry. Cell Microbiol 2004, 6(5):401-410.

3. Roop C, Hutchinson L, Johnson DC: A mutant herpes simplex virus type 1 unable to express glycoprotein $\mathrm{L}$ cannot enter cells, and its particles lack glycoprotein H. J Virol 1993, 67(4):2285-2297.

4. Geraghty RJ, Krummenacher C, Cohen GH, Eisenberg RJ, Spear PG: Entry of alphaherpesviruses mediated by poliovirus receptor-related protein 1 and poliovirus receptor. Science 1998, 280(5369):1618-1620.

5. Maclean CA: HSV Entry and Spread. Meth Mol Med 1998, 10:9-18.

6. Fernandez EJ, Lolis E: Structure, function, and inhibition of chemokines. Annu Rev Pharmacol Toxicol 2002, 42:469-499.

7. MacDonald MR, Li XY, Virgin HW: Late expression of a beta chemokine homolog by murine cytomegalovirus. J Virol 1997, 71(2):1671-1678.

8. Smith CA, Smith TD, Smolak PJ, Friend D, Hagen H, Gerhart M, Park L, Pickup DJ, Torrance D, Mohler K, et al: Poxvirus genomes encode a secreted, soluble protein that preferentially inhibits beta chemokine activity yet lacks sequence homology to known chemokine receptors. Virology 1997, 236(2):316-327.

9. Littman DR: Chemokine receptors: keys to AIDS pathogenesis? Cell 1998, 93(5):677-680. 
10. Murphy PM: Viral exploitation and subversion of the immune system through chemokine mimicry. Nat Immunol 2001, 2(2):116-122.

11. Boomker JM, de Leij LF, The TH, Harmsen MC: Viral chemokine-modulatory proteins: tools and targets. Cytokine Growth Factor Rev 2005, 16(1):91-103.

12. Wyrwicz $\mathrm{LS}$, Rychlewski $\mathrm{L}$ : Herpes glycoprotein $\mathrm{gL}$ is distantly related to chemokine receptor ligands. Antiviral Res 2007, 75(1):83-86.

13. Chowdary TK, Cairns TM, Atanasiu D, Cohen GH, Eisenberg RJ, Heldwein EE: Crystal structure of the conserved herpesvirus fusion regulator complex gH-gL. Nat Struct Mol Biol 2010, 17(7):882-888.

14. Matsuura $\mathrm{H}$, Kirschner $A N$, Longnecker R, Jardetzky TS: Crystal structure of the Epstein-Barr virus (EBV) glycoprotein $\mathrm{H} / \mathrm{glycoprotein} \mathrm{L}(\mathrm{gH} / \mathrm{gL})$ complex. Proc Natl Acad Sci USA 2010, 107(52):22641-22646.

15. Kaptein SJ, van Cleef KW, Gruijthuijsen YK, Beuken EV, van Buggenhout L, Beisser PS, Stassen FR, Bruggeman CA, Vink C: The r131 gene of rat cytomegalovirus encodes a proinflammatory CC chemokine homolog which is essential for the production of infectious virus in the salivary glands. Virus Genes 2004, 29(1):43-61.

16. Vomaske J, Denton M, Kreklywich C, Andoh T, Osborn JM, Chen D, Messaoudi I, Orloff SL, Streblow DN: Cytomegalovirus CC Chemokine Promotes Immune Cell Migration. J Virol 2012, 86(21):11833-11844.

17. Patrone M, Secchi M, Fiorina L, lerardi M, Milanesi G, Gallina A: Human cytomegalovirus UL130 protein promotes endothelial cell infection through a producer cell modification of the virion. J Virol 2005, 79(13):8361-8373.

18. Rajarathnam K, Sykes BD, Dewald B, Baggiolini M, Clark-Lewis I: Disulfide bridges in interleukin-8 probed using non-natural disulfide analogues: dissociation of roles in structure from function. Biochemistry 1999, 38(24):7653-7658.

19. Vink C, Beisser PS, Bruggeman CA: Molecular mimicry by cytomegaloviruses. Function of cytomegalovirus-encoded homologues of $\mathrm{G}$ protein-coupled receptors, $\mathrm{MHC}$ class I heavy chains and chemokines. Intervirology 1999, 42(5-6):342-349.

20. Altschul SF, Madden TL, Schaffer AA, Zhang J, Zhang Z, Miller W, Lipman DJ: Gapped BLAST and PSI-BLAST: a new generation of protein database search programs. Nucleic Acids Res 1997, 25(17):3389-3402.

21. Thompson JD, Gibson TJ, Higgins DG: Multiple sequence alignment using ClustalW and ClustalX. Current protocols in bioinformatics/editoral board, Andreas D Baxevanis [et al.]. 2002. Chapter 2:Unit 23.

22. McGuffin $L J$, Bryson $K$, Jones DT: The PSIPRED protein structure prediction server. Bioinformatics 2000, 16(4):404-405.

23. BioEdit: Biological sequence alignment editor for Win95/98/NT/2 KXP, Version 7.1.3. http://www.mbio.ncsu.edu/BioEdit/bioedit.html.

24. Sali A, Blundell TL: Comparative protein modeling by satisfaction of spatial restraints. J Mol Biol 1993, 234(3):779-815.

25. The PyMOL Molecular Graphics System, Version 0.99rc6. http://pymol.org/.

26. Saccoccio FM, Sauer AL, Cui X, Armstrong AE, el SE H, Johnson DC, Ryckman BJ, Klingelhutz AJ, Adler SP, McVoy MA: Peptides from cytomegalovirus UL130 and UL131 proteins induce high titer antibodies that block viral entry into mucosal epithelial cells. Vaccine 2011, 29(15):2705-2711.

27. Saccoccio FM, Gallagher MK, Adler SP, McVoy MA: Neutralizing activity of saliva against cytomegalovirus. Clin Vaccine Immunol: CVI 2011, 18(9):1536-1542.

doi:10.1186/1743-422X-10-1

Cite this article as: Malkowska et al: Alphaherpesvirinae and Gammaherpesvirinae glycoprotein L and CMV UL130 originate from chemokines. Virology Journal 2013 10:1.

\section{Submit your next manuscript to BioMed Central and take full advantage of:}

- Convenient online submission

- Thorough peer review

- No space constraints or color figure charges

- Immediate publication on acceptance

- Inclusion in PubMed, CAS, Scopus and Google Scholar

- Research which is freely available for redistribution 